\title{
CVChatbot: Um Chatbot para o Aplicativo Facebook Messenger Integrado ao AVA Moodle
}

\author{
${ }^{1}$ Paulo Dehon C. Cedro, ${ }^{2}$ Alexandre J. Carvalho Silva, ${ }^{3}$ Ana Carolina \\ Inocêncio, ${ }^{2}$ Cleber C. Castro, ${ }^{1}$ Heitor A. X. Costa, ${ }^{1}$ Paulo A. Parreira Júnior \\ ${ }^{1}$ Departamento de Ciência da Computação - Universidade Federal de Lavras (UFLA) \\ ${ }^{2}$ Diretoria de Educação a Distância - Universidade Federal de Lavras (UFLA) \\ Caixa Postal 3.037 - 37.200-000 - Lavras - MG - Brasil \\ ${ }^{3}$ Curso de Ciência da Computação - Universidade Federal de Jataí (UFJ) \\ Jataí, GO - Brasil \\ \{pdehon.cc, anacarolina.inocencio, clebercastrouai\}@gmail.com, \\ coord.reducdired.ufla.br, \{heitor, pauloa.junior\}@dcc.ufla.br
}

\begin{abstract}
This paper proposes an integration of the LMS Moodle with the Facebook Messenger app, in order to improve the process of communication among students and teachers in a virtual learning environment. Hence, a chatbot was developed for this app, called CVChatbot, which is able to send notifications to the users on modules created by the teachers in the virtual rooms of the LMS, as well as on messages posted in forums of these rooms. The developed chatbot was evaluated by 17 (seventeen) undergraduate students from a university and the results were considered promising.

Resumo. Este artigo propõe a integração do AVA Moodle com o aplicativo de troca de mensagens Facebook Messenger, com o intuito de aprimorar o processo de comunicação entre alunos e professores em um ambiente virtual de aprendizagem. Para isso, desenvolveu-se um chatbot para esse aplicativo, denominado CVChatbot, o qual é capaz de enviar notificações aos usuários sobre módulos criados pelos professores nas salas virtuais do AVA, bem como sobre mensagens postadas em fóruns dessas salas. O chatbot desenvolvido foi avaliado por 17 (dezessete) alunos de graduação de uma instituição de ensino superior e os resultados foram considerados promissores.
\end{abstract}

\section{Introdução}

Instituições educacionais, principalmente as de ensino superior, têm buscado meios nas Tecnologias de Informação e Comunicação (TIC) para otimizar a troca de informações entre professores e alunos para aprimorar o processo de ensino e aprendizagem. Um tipo de TIC utilizada com esse propósito são os Ambientes Virtuais de Aprendizagem (AVA). Um AVA é um software desenvolvido para auxiliar professores na promoção do ensino e aprendizagem de forma virtual [SEDIS, 2011]. O Moodle é um dos AVA mais utilizado no mundo, possuindo mais de 94 mil instalações (instâncias) em 234 países, mais de 14 milhões de cursos e 500 milhões de usuários inscritos [MOODLE, 2018]. 
VII Congresso Brasileiro de Informática na Educação (CBIE 2018)

Anais do XXIX Simpósio Brasileiro de Informática na Educação (SBIE 2018)

Campus Virtual é o nome dado à instância do Moodle, instalada na Universidade Federal de Lavras (UFLA). Por meio dela, professores, alunos e técnicosadministrativos têm à disposição recursos e possibilidades para viabilizar e otimizar o processo de ensino e aprendizagem no espaço institucional.

A quantidade de acessos diários de usuários únicos à Campus Virtual varia entre 2 mil a 4 mil, enquanto que a quantidade de usuários cadastrados na plataforma ultrapassa os 19 mil, distribuídos em 4.398 salas virtuais. Por meio de entrevistas realizadas com funcionários da DIRED (Diretoria de Educação a Distância), órgão da instituição que administra a Campus Virtual, percebeu-se que os alunos não têm o hábito de entrar nas salas virtuais da plataforma para checar as mensagens dos fóruns de suas disciplinas. Além disso, a caixa de entrada da conta de e-mail institucional dos alunos, para onde são direcionadas as mensagens, não é constantemente verificada. Isso foi percebido por causa de constantes reclamações de professores, alegando que seus alunos não têm recebido suas mensagens. Segundo os profissionais da DIRED, isto se deve, principalmente, a dois fatores: i) o advento da nova forma de interação entre indivíduos, proporcionada pelos dispositivos móveis, especialmente por meio de aplicativos de troca de mensagens (e.g., Telegram, WhatsApp e Facebook Messenger); e ii) o fato de o e-mail institucional não ser a conta de $e$-mail principal dos discentes.

Nos últimos anos, aplicativos de troca de mensagens tornaram-se o meio mais utilizado para se comunicar por meio de dispositivos móveis [CHURCH \& DE OLIVEIRA, 2013]. Tais aplicativos têm trazido maior praticidade quanto à divulgação e à obtenção de informações das mais diversas fontes, desviando o fluxo de uso dos tradicionais AVA e de outros tipos de serviços, como o e-mail e websites. Logo, para AVA continuarem eficazes, faz-se necessário que eles sigam o avanço da tecnologia, adaptando-se aos novos perfis de interação entre usuários [TRIFONOVA; GEORGIEVA \& RONCHETTI, 2006; CHANG \& SHEU, 2002].

Integrar AVA com esse tipo de aplicativo pode ter efeitos positivos no processo de ensino e aprendizagem, pois podem possibilitar melhor propagação das informações disponibilizadas nesses ambientes [HRASTINSKI, 2006]. Na UFLA; isso acontece, mesmo que de forma não institucionalizada. Por exemplo, alguns professores preferem enviar mensagens aos seus alunos via Facebook, pois sabem que eles terão acesso a elas mais rapidamente do que se elas fossem enviadas via Campus Virtual.

Uma forma de garantir a interação aluno/professor em aplicativos de troca de mensagens é por meio da criação de grupos particulares nesses aplicativos, geralmente criados e administrados pelo próprio professor da disciplina. Porém, essa é uma estratégia limitada para o uso institucional, pois as informações trocadas entre alunos e professores só ficam registradas em suas contas pessoais e dispositivos móveis particulares. Além disso, tal estratégia exige retrabalho por parte do professor, uma vez que ele teria que compartilhar tudo que foi publicado no AVA nos grupos criados. Outra limitação é o esforço necessário para a criação de grupos para inclusão dos alunos, bem como para envio dos conteúdos nesses grupos.

Neste trabalho, acredita-se que uma estratégia interessante para aproximar alunos e professores, no contexto de uso de AVA, é enviando notificações das ações realizadas nas salas virtuais para os aplicativos de troca de mensagens dos alunos, por meio de um chatbot. Chatbots são scripts desenvolvidos para serem executados em 
VII Congresso Brasileiro de Informática na Educação (CBIE 2018)

Anais do XXIX Simpósio Brasileiro de Informática na Educação (SBIE 2018)

aplicativos de troca de mensagens para realizarem operações específicas para seus usuários (mais informações sobre chatbots são apresentadas na Seção 2). Neste sentido, o principal objetivo é apresentar o CVChatbot, um chatbot desenvolvido para o aplicativo Facebook Messenger que se integra ao Moodle, mais especificamente à Campus Virtual. O chatbot desenvolvido foi avaliado por alunos da UFLA e como resultados, notou-se que a maioria deles considerou o CVChatbot um recurso útil e que pode aperfeiçoar a comunicação entre alunos e professores ao longo do curso.

Este trabalho encontra-se organizado da seguinte forma. Na Seção 2, são discutidos alguns conceitos importantes para seu entendimento. Na Seção 3, são apresentados a arquitetura e o funcionamento do CVChatbot. Na Seção 4, é mostrada a avaliação realizada com o CVChatbot, bem como são discutidos os principais resultados obtidos. Na Seção 5, são apresentadas considerações finais e trabalhos futuros.

\section{AVAs, Aplicativos de Troca de Mensagens \& Chatbots}

A maior parte das instituições de ensino estão incorporando TIC no contexto educacional para assistir os cursos e as disciplinas presenciais, semipresenciais ou à distância [PENTERICH, 2014]. AVA são tecnologias, cujo intuito é fornecer uma plataforma para troca de informações entre alunos e professores, na qual há melhor controle das tarefas realizadas pelos alunos e do acompanhamento dos professores a respeito do interesse dos alunos nos materiais disponibilizados. AVA podem ser definidos como sistemas de ensino e aprendizagem integrados e abrangentes capazes de promover o interesse do aluno, complementando a interação em sala de aula, do professor e do aluno, em um ambiente virtual independente e flexível e permitindo ao aluno acessar as informações disponibilizadas neles de qualquer lugar e em qualquer horário [VAZ; ZANELLA \& ANDRADE, 2010].

Entre AVAs mais utilizados, estão [SILVA, 2014]: Moodle, TelEduc, AulaNet, Claroline, BlackBoard e E-Proinfo. Dentre esses, o Moodle é um dos AVA mais utilizado por ser um software de código aberto, livre e gratuito. Após uma pesquisa comparativa [SILVA, 2014] entre os AVAs de código livre (Moodle, Claroline e Amadeus), concluiu-se que o Moodle tem vantagens sobre os demais, por causa da quantidade de funções e da quantidade de recursos disponibilizados para uso pedagógico. Além disso, ele foi considerado o mais adequado por (i) ser mais completo, em decorrência dos recursos que apresenta, (ii) permitir avaliação por meio de notas, (iii) permitir o uso de repositórios externos, (v) possuir vasta gama de opções de plugins para introdução de novos recursos ao ambiente e (iv) permitir a customização de layout, de acordo com as características dos usuários.

TIC de massa, tais como redes sociais e aplicativos de troca de mensagens, têm participado cada vez mais do cotidiano dos indivíduos [PINOCHET, 2014]. Aplicativos de troca de mensagens são sistemas de software para dispositivos móveis (em alguns casos, há também versões para $W e b$ ), nos quais os usuários podem se comunicar com seus contatos, por meio de mensagens de texto, voz, vídeos, fotos, entre outros. A utilização desse tipo de aplicativo cresceu significantemente com a era dos dispositivos móveis, por ser um meio de comunicação barato e prático [KANTAR.COM, 2017]. Entre os aplicativos de troca de mensagens mais populares no mundo, estão WhatsApp, Telegram, Facebook Messenger, Skype e Hangouts, sendo o mais utilizado WhatsApp. 
VII Congresso Brasileiro de Informática na Educação (CBIE 2018)

Anais do XXIX Simpósio Brasileiro de Informática na Educação (SBIE 2018)

WhatsApp está presente em mais de 85\% dos dispositivos móveis, Facebook Messenger, com $74 \%$ do mercado, e WeChat, está presente em mais de $57 \%$ dos dispositivos móveis existentes [STATISTA.COM, 2018].

Chatbots são scripts executados sobre a plataforma dos aplicativos de troca de mensagens, que realizam funções específicas. Existem chatbots relacionados às diversas áreas, como educação, entretenimento e pesquisa [TAKE.COM, 2016]. Por exemplo, "ImageBot" é um chatbot para o aplicativo Telegram, cujo objetivo é buscar imagens na Web por meio das palavras-chave enviadas pelo usuário. "Max", disponível para alguns dos aplicativos de troca de mensagens, tais como Skype e Telegram, é capaz de agendar eventos em geral, armazenando o nome das pessoas que comparecerão, o horário e o local do evento, dentre outras informações. O ponto forte dos chatbots é eles oferecerem um serviço embutido nas ferramentas mais utilizadas no cotidiano dos indivíduos, i.e., os aplicativos de troca de mensagem. Além disso, para utilizar um chatbot, não é necessário fazer download de outros aplicativos, reduzindo-se o espaço ocupado no dispositivo móvel do usuário, bem como a necessidade de familiarização com a interface desses aplicativos. Para desenvolver um chatbot para um aplicativo de troca de mensagens, é necessário que esse aplicativo forneça uma API (Application Programming Interface) de integração para coletar os dados de entrada dos usuários e enviar informações aos usuários. Aplicativos como Skype, Facebook Messenger e Telegram, possuem essas APIs. Na Seção 3, são apresentados, com mais detalhes, a arquitetura e o funcionamento de um chatbot para o aplicativo Facebook Messenger.

\section{CVChatbot}

Antes de iniciar a integração da Campus Virtual com aplicativos de troca de mensagens, foi realizada uma pesquisa de opinião para (i) conhecer as principais formas de acesso à Campus Virtual por parte dos alunos, (ii) saber a frequência com que ocorrem os acessos, (iii) entender as principais limitações enfrentadas pelos alunos ao tentarem acessar a Campus Virtual e as formas de minimizá-las e (iv) conhecer a proporção de alunos com dispositivos móveis e quais são os aplicativos de troca de mensagens mais utilizados por eles. Para isso, foi criado um questionário eletrônico, disponibilizado a toda comunidade acadêmica da UFLA por um período de 15 dias. Algumas questões presentes nesse questionário, elaboradas com o auxílio de funcionários e de professores envolvidos com a DIRED, estão na Tabela 1. O questionário completo pode ser encontrado em [Cedro, 2018], o qual recebeu 254 respostas. As principais observações obtidas a partir dessas respostas são descritas a seguir.

Tabela 1. Algumas questões utilizadas para análise de viabilidade.

\begin{tabular}{|l|l|}
\hline \multicolumn{2}{|c|}{ Questão } \\
\hline $\begin{array}{l}\text { Pocê possui dispositives móveis, tais como smartphonestas ou } \\
\text { tablets? }\end{array}$ & $\begin{array}{l}\text { () Sim, apenas smartphone; ( ) Sim, apenas tablet; ( ) Sim, os } \\
\text { dois. () Não }\end{array}$ \\
\hline $\begin{array}{l}\text { Qual(is) dos aplicativos de troca de mensagens a seguir você } \\
\text { possui instalado em seu(s) dispositivo(s) móvel(is)? }\end{array}$ & $\begin{array}{l}\text { () WhatsApp; ( ) Telegram; ( ) Skype; ( ) Facebook Messenger; } \\
\text { () Nenhum; ( ) Outro. Especifique: }\end{array}$ \\
\hline $\begin{array}{l}\text { Quando você acessa a Campus Virtual, na maioria das vezes, } \\
\text { é para.... }\end{array}$ & $\begin{array}{l}\text { () Ler/enviar mensagens do/no Mural de Recados; ( ) Baixar } \\
\text { slides/material das aulas; ( ) Enviar mensagens ao professor; ( ) } \\
\text { Outro. Especifique: }\end{array}$ \\
\hline
\end{tabular}

Aproximadamente, 91\% dos respondentes afirmaram possuir tablets ou smartphones. Os aplicativos de troca de mensagens mais utilizados são WhatsApp (95,3\%) e Facebook Messenger (76,4\%). Em torno de 94\% dos respondentes utilizam 
VII Congresso Brasileiro de Informática na Educação (CBIE 2018)

Anais do XXIX Simpósio Brasileiro de Informática na Educação (SBIE 2018)

os aplicativos de troca de mensagens todos os dias. Por volta de $87 \%$ dos respondentes utilizam o computador para acessar a Campus Virtual.

A maioria dos respondentes utiliza a Campus Virtual para fazer download de slides $(79,1 \%)$ e listas de exercícios (69,3\%). Além disso, 47,2\% utilizam para ler/enviar mensagens no mural de recados. Após a Campus Virtual, a maior fonte de consulta de informações das disciplinas $(43,3 \%)$ são grupos em aplicativos de troca de mensagens e redes sociais, criados por professores ou alunos. A maioria dos respondentes $(87,4 \%)$ prefere receber avisos sobre suas disciplinas via dispositivos móveis. Contudo, segundo os respondentes, os maiores problemas para aceitação da instalação de um aplicativo móvel para obtenção dessas informações são o consumo de memória que eles exigem $(48,4 \%)$ e a quantidade limitada de funções disponíveis $(33,1 \%)$, totalizando $81,5 \%$ das respostas. Por fim, $81,9 \%$ dos respondentes disseram não enxergar problemas em adicionar mais um contato em seus dispositivos de troca de mensagens para obter informações de suas disciplinas.

Com base na pesquisa realizada, notou-se que a integração da Campus Virtual com aplicativos de troca de mensagens pareceu ser bem aceita pela comunidade acadêmica. Apesar de não ser o aplicativo mais utilizado pelos usuários da UFLA, o Facebook Messenger está na segunda colocação de aplicativo mais utilizado e possui uma API que permite o desenvolvimento de chatbots, ao contrário de seu concorrente, o WhatsApp. Dessa forma, neste trabalho, optou-se pelo desenvolvimento de um chatbot para o aplicativo Facebook Messenger.

\subsection{Configuração e Funcionamento do CVChatbot}

Nesta seção, são abordados os passos necessários para os usuários (alunos e professores) conseguirem realizar a integração entre suas contas da Campus Virtual e do Facebook Messenger, via CVChatbot. Além disso, são apresentadas as principais funções do chatbot.

Inicialmente, o aluno deve acessar a página do CVChatbot no Facebook e iniciar uma conversa, clicando no botão "Começar" no chat da página. O link dessa página pode ser amplamente divulgado à comunidade acadêmica, por meio da Assessoria de Comunicação da instituição ou por meio de um aviso colocado na página principal do AVA. Após a conversa ser iniciada, toda interação entre usuário/chatbot ocorrerá por meio de menus de opções. Inicialmente, o aluno deve realizar acesso ao AVA Campus Virtual. Para agilizar o processo de interação, o CVChatbot envia uma mensagem com um link de acesso ao AVA e, logo após, envia uma mensagem de confirmação, questionando se o usuário conseguiu ou não se autenticar no Campus Virtual. Caso o usuário não tenha conseguido acesso, o chatbot envia uma mensagem para o suporte da DIRED para regularização da situação.

Uma vez tendo conseguido acesso, uma mensagem com link para a página "Modificar Perfil", da Campus Virtual, será enviada ao usuário (Figura 1 - A). Juntamente com essa mensagem, são enviadas algumas orientações sobre as alterações a serem feitas no perfil do usuário. Ele deve copiar o código encaminhado nessa mensagem (omitido na imagem para garantia de privacidade) e colocá-lo no campo "Código do Facebook Messenger", da página de atualização de perfil do AVA. Além 
VII Congresso Brasileiro de Informática na Educação (CBIE 2018)

Anais do XXIX Simpósio Brasileiro de Informática na Educação (SBIE 2018)

disso, ele deve aceitar o termo de condições de uso do chatbot. O termo de condições de uso do CVChatbot, disponibilizado pela UFLA, pode ser encontrado em [Cedro, 2018].

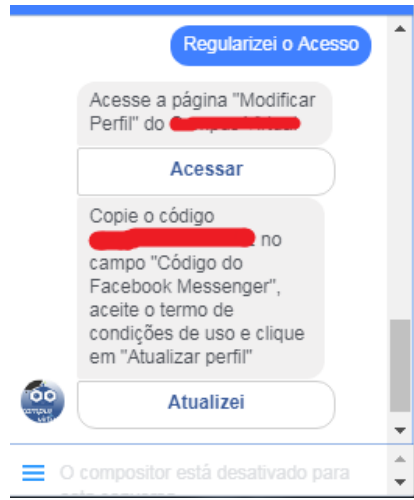

(A)

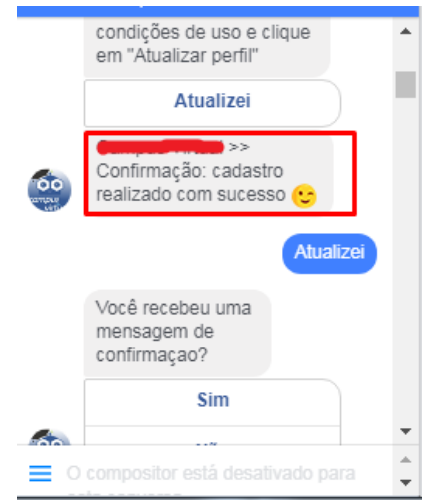

(B)

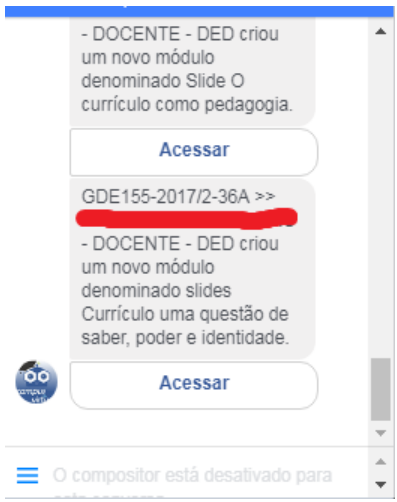

(C)

Figura 1. Interface de uso do CVChatbot.

Se tudo estiver correto, o usuário recebe, via chatbot, uma mensagem de sucesso (Figura 1 - B). Após receber essa mensagem, o CVChatbot está pronto para uso, notificando o usuário dos diversos eventos que ocorrem no Campus Virtual. Concluída a configuração por parte do usuário, os módulos posteriormente cadastrados pelos professores nas salas virtuais da Campus Virtual correspondentes às suas disciplinas (por exemplo, questionários, arquivos e fóruns) são automaticamente notificados aos usuários interessados via chatbot. Tal notificação consiste de uma mensagem no aplicativo Facebook Messenger, contendo: i) o nome de quem criou o evento (em geral, o professor da disciplina ou algum monitor com permissão para criar módulos); (ii) o nome da disciplina à qual se refere o módulo (isso é útil, pois o usuário que recebeu a mensagem pode estar cadastrado em várias disciplinas em um mesmo semestre); (iii) o nome do módulo criado (escolhido pelo usuário que o criou); e (iv) um link de acesso a esse módulo no Campus Virtual. Um exemplo de mensagem de notificação pode ser visto na Figura 1 - C).

Além de notificações sobre a criação de módulos, o CVChatbot notifica o usuário sobre mensagens enviadas nos fóruns assinados por ele no AVA (diz-se que um fórum é assinado, quando o usuário opta por receber mensagens postadas naquele fórum). No caso da Campus Virtual, toda sala virtual é composta de um fórum, denominado "Mural de Recados", no qual todo participante da sala torna-se, automaticamente, um assinante. A notificação de mensagens postadas no fórum é composta por: i) o nome de quem enviou a mensagem; ii) o nome da disciplina à qual se refere o fórum em que a mensagem foi postada; e iii) um link de acesso a mensagem no Campus Virtual.

\subsection{Arquitetura do CVChatbot}

Na Figura 2 - A, é representada, sucintamente, a arquitetura do CVChatbot. A solução de integração entre a Campus Virtual e o aplicativo Facebook Messenger, a saber CVChatbot, é composta por duas partes: i) um chatbot para o aplicativo Facebook Messenger; e ii) um plug-in para o Moodle denominado FBNotifier (FaceBook Notifier). A existência desse plug-in é fundamental para integração da Campus Virtual 
VII Congresso Brasileiro de Informática na Educação (CBIE 2018)

Anais do XXIX Simpósio Brasileiro de Informática na Educação (SBIE 2018)

com o aplicativo Facebook Messenger, pois ele recebe as notificações ocorridas no AVA e as encaminha para o chatbot via API do Facebook Messenger.

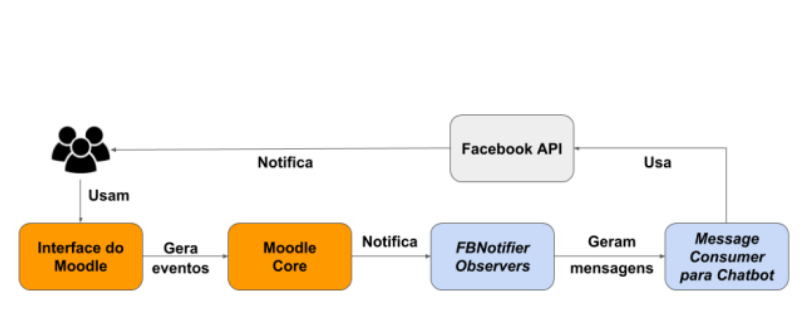

(A)

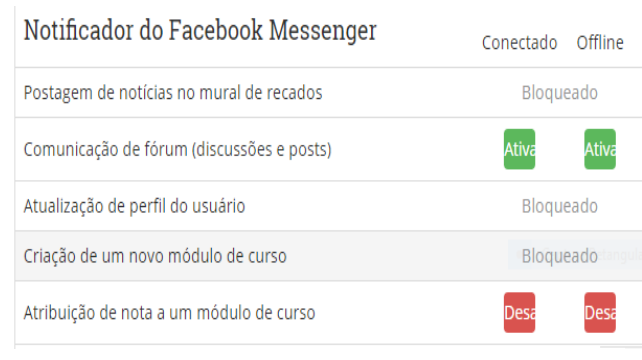

(B)

Figura 2. Arquitetura do CVChatbot (A) e painel de configuração de preferências de recebimento de mensagens $(B)$.

Ainda na Figura 2 - A, é apresentada como essas duas partes se comunicam para proporcionar o funcionamento do CVChatbot. Inicialmente, usuários interagem com a interface do Moodle, mais especificamente da Campus Virtual, por meio de sua interface gráfica com o usuário. As interações realizadas por esses usuários geram eventos, os quais são tratados pelo código core do Moodle. O plug-in FBNotifier, uma vez instalado na plataforma Moodle, registra observadores para determinados eventos de interesse do CVChatbot, por exemplo, a criação de um módulo em uma sala virtual e a postagem de uma notícia em um fórum. Assim, quando tais eventos de interesse ocorrem, os observadores do plug-in são notificados pelo código core do Moodle e mensagens são enviadas aos aplicativos Facebook Messenger dos usuários interessados por aquela mensagem via API do Facebook. Por exemplo, se o professor de uma sala virtual criar um módulo denominado "Slides da aula de Padrões de Projeto", todos os alunos matriculados naquela sala (apenas estes) recebem uma mensagem em seus aplicativos (Figura 1 - C).

Outro ponto importante a ser ressaltado é o fato de o FBNotifier ser do tipo Message Consumer, ou seja, ele representa um tipo de "alvo", para o qual mensagens e notificações do AVA podem ser enviadas. O Moodle possui diversos tipos de Message Consumers, tais como e-mail e SMS. Uma preocupação existente com a integração da Campus Virtual com o Facebook Messenger é não sobrecarregar alunos e professores com demasiadas mensagens e notificações enviadas pelo chatbot. Contudo, quando se constrói um plug-in do tipo Message Consumer, o Moodle disponibiliza uma interface aos usuários para a configuração das preferências de recebimento de notificações (Figura 2 - B). Por exemplo, nessa figura, pode-se notar que o usuário optou por receber notificações (Opção "Ativado") de mensagens postadas em fóruns, mas não de atribuições de nota a módulos de curso (Opção "Desativado"). As opções que aparecem com o status "Bloqueado" referem-se às configurações feitas pelo administrador da plataforma Moodle e que não podem ser alteradas pelo usuário.

Por questão de limitação de espaço, não é possível descrever todos os passos da construção e instalação do chatbot, bem como do plug-in FBNotifier. Contudo, esses passos estão documentados e acessíveis em [Cedro, 2018]. Além disso, cabe ressaltar que todo código do CVChatbot é livre e está disponível para download em [DIRED/UFLA, 2018]. 
VII Congresso Brasileiro de Informática na Educação (CBIE 2018)

Anais do XXIX Simpósio Brasileiro de Informática na Educação (SBIE 2018)

\section{Avaliação do CVChatbot}

O CVChatbot foi avaliado por um grupo de alunos de graduação da UFLA, para conhecer a satisfação dos usuários e os benefícios que ele pode trazer para a comunidade acadêmica, em particular, para os alunos. Para isso, os seguintes procedimentos foram realizados:

1. O CVChatbot foi apresentado a um grupo de 70 alunos dos cursos de Ciência da Computação e Engenharia de Controle e Automação, dentre os quais, 17 deles aceitaram voluntariamente participar da avaliação; e

2. A avaliação ocorreu por um período de 15 dias. Como atividades, os alunos tiveram que integrar suas contas do Facebook Messenger com as da Campus Virtual (conforme descrito na Seção 3.1) e receber/visualizar as mensagens encaminhadas pelo professor da disciplina em seus dispositivos móveis, a partir do aplicativo Facebook Messenger.

Após o período de avaliação, foi aplicado um questionário eletrônico aos alunos (Tabela 2). As questões presentes nesse questionário foram elaboradas com o auxílio de funcionários e professores envolvidos com a DIRED. Para cada questão, os alunos deviam escolher uma dentre as seguintes opções: Discordo totalmente, Discordo parcialmente, Neutro, Concordo parcialmente e Concordo totalmente. As respostas para essas questões são apresentadas na Tabela 3.

Tabela 2. Questões utilizadas na avaliação do CVChatbot.

\begin{tabular}{|l|l|}
\hline 1. Eu gostei de usar o CVChatbot. & $\begin{array}{l}\text { 7. Usar o CVChatbot pode aumentar meu desempenho no } \\
\text { processo de ensino e aprendizado. }\end{array}$ \\
\hline 2. A configuração (integração) do CVChatbot é simples. & $\begin{array}{l}\text { 8. Usar o CVChatbot pode aumentar meu desempenho quanto } \\
\text { ao planejamento das minhas atividades acadêmicas. }\end{array}$ \\
\hline 3. Usar o CVChatbot é uma boa ideia. & $\begin{array}{l}\text { 9. O CVChatbot apresenta notificações úteis sobre as } \\
\text { disciplinas em que estou matriculado. }\end{array}$ \\
\hline 4. No CVChatbot, é fácil encontrar a informação que desejo. & $\begin{array}{l}\text { 10. O CVChatbot produz os resultados que espero de uma } \\
\text { ferramenta de suporte à comunicação entre professores e } \\
\text { alunos. }\end{array}$ \\
\hline 5. O CVChatbot possui visual adequado. & 11. Eu pretendo integrar o CVChatbot a minha vida acadêmica. \\
\hline $\begin{array}{l}\text { 6. O CVChatbot é útil no processo de comunicação entre } \\
\text { professores e alunos. }\end{array}$ & 12. Eu recomendarei o uso do CVChatbot. \\
\hline
\end{tabular}

Quanto às respostas obtidas no questionário de avaliação, pode-se notar a aceitação para utilizar o CVChatbot como recurso complementar, visto que $77,8 \%$ dos alunos gostaram de utilizar a aplicação e $83,3 \%$ acham CVChatbot uma boa ideia. Porém, alguns pontos precisam ser melhorados, por exemplo, com relação às informações para realizar a configuração do CVChatbot. Apesar de 72,2\% acharem o tutorial de instalação claro e útil, apenas 55,6\% concordaram com a facilidade de obter informações para realizar a instalação. É importante observar que o CVChatbot não foi desenvolvido para substituir as funções do Moodle, mas para otimizar as funções de propagação de informação entre professores e alunos. Pode-se observar que tal otimização foi bem recebida pelos alunos, uma vez que $77,8 \%$ gostaram das notificações do CVChatbot e $72,2 \%$ o consideraram útil na comunicação entre alunos e professores. Além disso, muitos alunos acreditam que o CVChatbot pode melhorar o seu desempenho acadêmico e o planejamento das atividades universitárias.

Além dessas questões, os alunos deviam responder a três outras questões abertas: i) qual(is) fator(es) lhe(s) faz(em) usar o CVChatbot?; ii) Qual(is) fator(es) lhe(s) 
VII Congresso Brasileiro de Informática na Educação (CBIE 2018)

Anais do XXIX Simpósio Brasileiro de Informática na Educação (SBIE 2018)

impede(m) de usar o CVChatbot?; e iii) Caso queira, aponte críticas/sugestões (por exemplo, novos tipos de notificações) sobre o CVChatbot. Com relação às respostas para essas questões, a maioria dos respondentes disse que a praticidade e a facilidade de acesso são o que os fazem usar o CVChatbot. Quanto aos fatores que impedem os usuários de usarem o CVChatbot, os respondentes disseram que não identificaram tais fatores. Como críticas e sugestões, os respondentes disseram esperar uma versão do chatbot para o aplicativo WhatsApp. Por enquanto, essa é uma limitação técnica, pois o WhatsApp não disponibiliza oficialmente uma API para construção de chatbots.

Tabela 3. Respostas das questões objetivas da avaliação do CVChatbot.

\begin{tabular}{|c|c|c|c|c|c|}
\hline Questão & $\begin{array}{l}\text { Concordo } \\
\text { Totalmente }\end{array}$ & $\begin{array}{c}\text { Concordo } \\
\text { Parcialmente }\end{array}$ & Neutro & $\begin{array}{c}\text { Discordo } \\
\text { Parcialmente }\end{array}$ & $\begin{array}{c}\text { Discordo } \\
\text { Totalmente }\end{array}$ \\
\hline 1 & $77,8 \%$ & $11,1 \%$ & $11,1 \%$ & $0 \%$ & $0 \%$ \\
\hline 2 & $72,2 \%$ & $11,1 \%$ & $16,7 \%$ & $0 \%$ & $0 \%$ \\
\hline 3 & $83,3 \%$ & $11,1 \%$ & $5,6 \%$ & $0 \%$ & $0 \%$ \\
\hline 4 & $55,6 \%$ & $22,2 \%$ & $16,7 \%$ & $5,6 \%$ & $0 \%$ \\
\hline 5 & $55,6 \%$ & $16,7 \%$ & $16,7 \%$ & $11,1 \%$ & $0 \%$ \\
\hline 6 & $72,2 \%$ & $16,7 \%$ & $11,1 \%$ & $0 \%$ & $0 \%$ \\
\hline 7 & $72,2 \%$ & $11,1 \%$ & $16,7 \%$ & $0 \%$ & $0 \%$ \\
\hline 8 & $72,2 \%$ & $16,7 \%$ & $11,1 \%$ & $0 \%$ & $0 \%$ \\
\hline 9 & $77,8 \%$ & $16,7 \%$ & $5,6 \%$ & $0 \%$ & $0 \%$ \\
\hline 10 & $83,3 \%$ & $11,1 \%$ & $5,6 \%$ & $0 \%$ & $0 \%$ \\
\hline 11 & $55,6 \%$ & $27,8 \%$ & $16,7 \%$ & $0 \%$ & $0 \%$ \\
\hline 12 & $66,7 \%$ & $11,1 \%$ & $22,2 \%$ & $0 \%$ & $0 \%$ \\
\hline
\end{tabular}

É importante ressaltar que o questionário avaliativo sobre o CVChatbot foi respondido por uma amostra pouco significativa, pois, além da baixa quantidade de participantes (apenas 17), eles pertenciam a apenas dois cursos de graduação de uma instituição de ensino superior. Por serem alunos de cursos relacionados à área de Computação, eles estão mais habituados com a utilização de tecnologias do que alunos de outros cursos, o que pode ter influenciado a avaliação do chatbot quanto à sua facilidade de uso. Para mitigar tais ameaças, pretende-se replicar o experimento com outros alunos da instituição, contemplando mais participantes de diferentes cursos. Além disso, pretende-se realizar uma análise por perfil de usuário, considerando o curso ao qual ele está vinculado, sua idade, entre outras informações.

\section{Considerações Finais}

O uso de dispositivos móveis e de aplicativos de troca de mensagens tem se tornado cada vez mais comum nos dias atuais. Segundo pesquisa realizada com 254 alunos de graduação da UFLA, notou-se que aproximadamente $94 \%$ dos estudantes acessam esses aplicativos diariamente. Por causa do frequente acesso, a maioria dos respondentes dessa pesquisa $(87,4 \%)$ afirmou preferir receber avisos sobre suas disciplinas via dispositivos móveis.

Nesse contexto, neste trabalho, foi proposta uma ferramenta para a integração do Moodle, mais especificamente da instância deste AVA instalada na UFLA, denominada Campus Virtual, com o aplicativo de troca de mensagens Facebook Messenger, via chatbot. Com essa integração, denominada CVChatbot, espera-se maior aproximação entre professores e alunos e entre os próprios alunos da instituição, o que pode favorecer o processo de ensino e aprendizagem. Além disso, espera-se institucionalizar uma prática que vem sendo realizada na instituição de ensino: criação de grupos em 
VII Congresso Brasileiro de Informática na Educação (CBIE 2018)

Anais do XXIX Simpósio Brasileiro de Informática na Educação (SBIE 2018)

aplicativos de troca para mensagens para divulgação de notícias e de arquivos relacionados às disciplinas.

Como propostas de trabalho futuro, têm-se: i) disponibilizar o CVChatbot para toda a comunidade acadêmica da UFLA; ii) facilitar o processo de configuração do CVChatbot por parte dos alunos para aumentar a sua adesão; iii) propor novas funções para o CVChatbot, como a possibilidade de o aluno enviar mensagens em fóruns de disciplina ou para algum usuário específico, a partir do CVChatbot; iv) aprimorar a avaliação das funções do CVChatbot para obter resultados mais robustos sobre as vantagens desse tipo integração; e v) expandir a integração para outros aplicativos, tais como Telegram, Skype e WhatsApp.

\section{Referências}

Cedro, P. D. C. CVChatbot: Um chatbot para o aplicativo Facebook Messenger integrado ao Ambiente Virtual de Aprendizagem Campus Virtual. Monografia de graduação. DCC/UFLA. 2018.

Chang, C.; Sheu, J. (2002). Design and Implementation of Ad Hoc Classroom and eSchoolbag Systems for Ubiquitous Learning. In: 1st IEEE International Workshop on Wireless and Mobile Technologies in Education, Växjö, Sweden, p. 8-14, Agosto, 2002.

Church, K.; de Oliveira, R. (2013). What's up with WhatsApp? Comparing mobile instant messaging behaviors with traditional SMS. Proceedings of the 15th international conference on human-computer interaction with mobile devices and services (pp. 352-361). Munich, Germany: ACM (August 27-30, 2013).

DIRED/UFLA. Página da DIRED/UFLA no Github. Disponível em: https:/github.com/dired-ufla/. Acessado em: Set/2018.

Hrastinski, S. (2006). Introducing an informal synchronous medium in a distance learning course: How is participation affected? Internet and Higher Education, 9(2), 117-131.

Kantar.com. Troca de mensagens instantâneas é um dos principais usos do celular no Brasil. Disponível em: https://br.kantar.com/tecnologia/m\%C3\%B3vel/2017/troca-de-mensagens-instant $\% \mathrm{C} 3 \% \mathrm{~A} 2$ neas\%C3\%A9-um-dos-principais-usos-do-celular-entre-brasileiros-comtech/. Acessado em: Jun/2018.

MOODLE. "Moodle statistics". Disponível em: < https://moodle.net/stats/ > . Acessado em: Jun/2018.

Pinochet, L. H. C. (2014). Tecn. da Informação e Comunicação - 1 ed. - Rio de Janeiro: Elsevier, 2014.

Penterich, E. Ambientes virtuais de aprendizagem, 2014. Disponível em: http://portal.metodista.br/atualiza/conteudo/material-de-apoio/didatico-pedagogico/livros/sala-de-aulae-tecnologias/cap05.pdf. Acessado em: Jun/2018.

SEDIS. “O que é um Ambiente Virtual”, 2011. Disponível em: http://www.sedis.ufrn.br/index.php/201107-07-10-32-54/o-que-e. Acessado em: Jun/2018.

SILVA, A. J. C. (2014). Desenvolvimento de uma comunidade virtual para a inserção da metodologia blended learning na educação básica - Lavras: UFLA, 2014. 135p.: il.

STATISTA.COM (2018). Disponível em: https:/www.statista.com/statistics/258749/most-popularglobal-mobile-messenger-apps/. Acessado em: Jun/2018.

TAKE.COM. Afinal, o que é um chatbot? (2016). Disponível em: http://chatbotsbrasil.take.net/afinal-oque-e-um-chatbot/. Acessado em: Jun/2018.

Trifonova, A.; Georgieva, E.; Ronchetti, M. (2006). Determining Students' Readiness for Mobile Learning. In: 5th Int. Conference on E-ACTIVITIES, Venice, Italy, p. 84-89, November, 2006.

Vaz, D.; Zanella, R.; Andrade, S. (2010). Ambientes Virtuais: Uma Nova Ferramenta de Ensino. Revista iTEC - Vol. I, No 1, Dez. 2010.BASTOS, L. da R. et al. Manual para a elaboração de projetos e relatórios de pesquisas, teses, dissertações e monografias. 6. ed. Rio de Janeiro: LTC, 2003. 